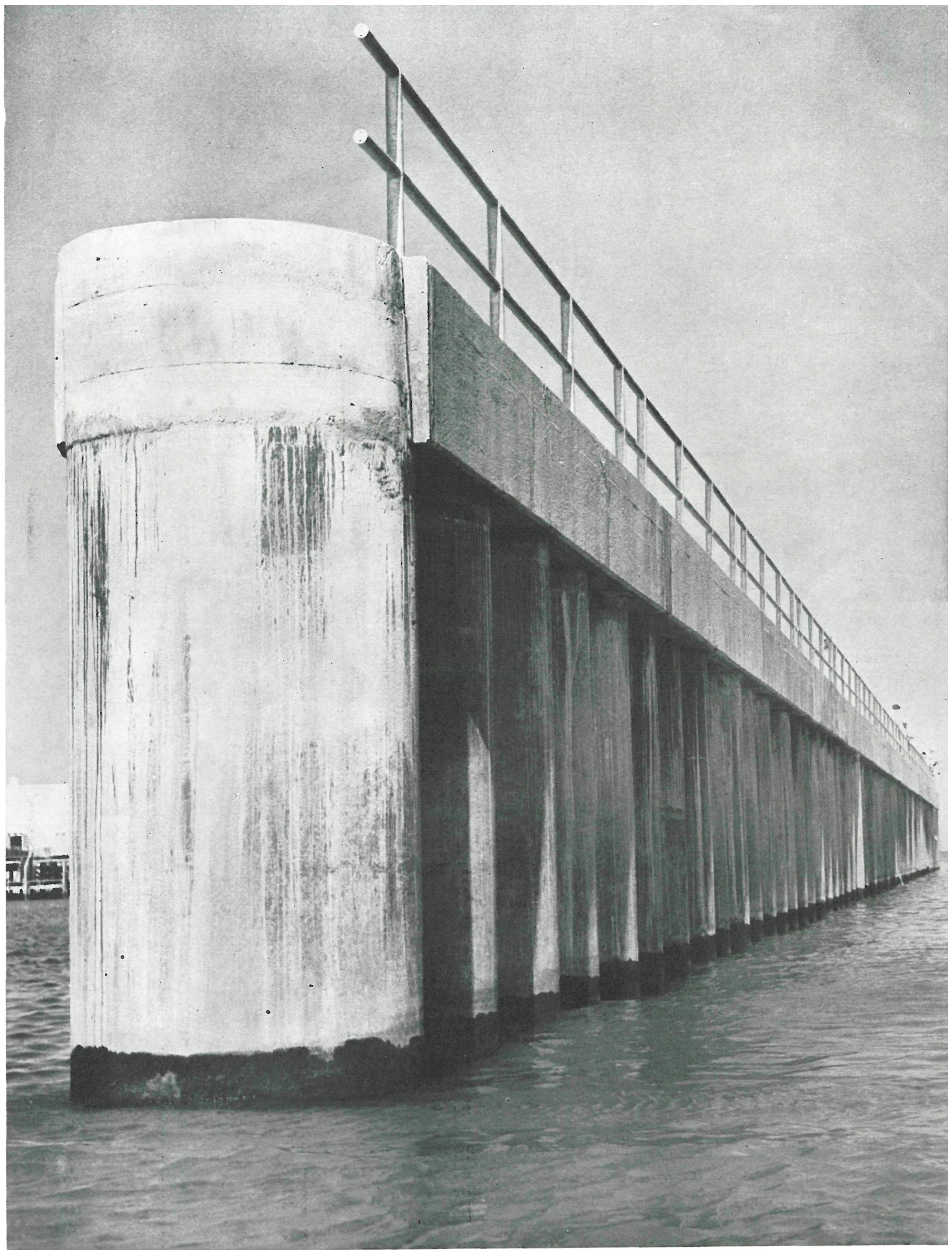

\title{
rompeolas de hormigón pretensado
}




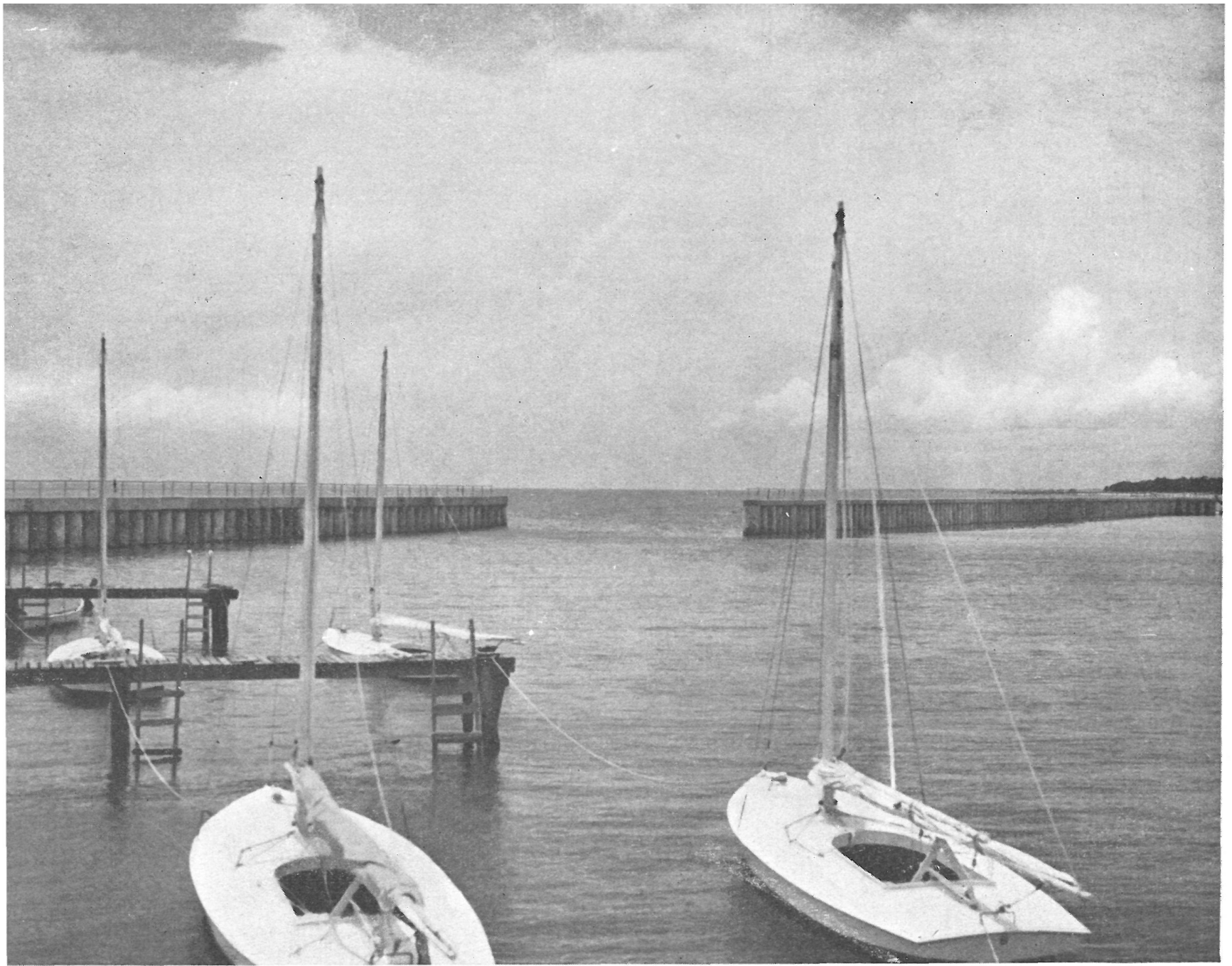

$558 \cdot 15$

Desde hace unos doce años, fecha en que se construyó en los Estados Unidos la primera estructura de hormigón pretensado, han sido más y distintas las aplicaciones posteriores que de esta técnica moderna se han venido realizando en dicho país. Todas las obras realizadas siguiendo estos procedimientos se han controlado estrechamente para conocer de cerca los resultados obtenidos en las múltiples y variadas aplicaciones, con objeto de adoptarlas en las distintas especialidades de la ingeniería civil, de entre las cuales, la parte maritima es la que más directamente se refiere a este trabajo.

Un ejemplo particular de las aplicaciones del hormigón pretensado a la construcción de obras marítimas lo constituye el rompeolas construído en el Golfo de Méjico, en la costa de la desembocadura del río Mississippi y en las proximidades de un pueblecito llamado Pass Christian, donde se acaba de terminar un rompeolas formado por cilindros pretensados yuxtapuestos coronados con una losa prefabricada de hormigón. El espacio entre cilindros se cerró con madera creosotada.

Es de notar que, en el concurso para la ejecución de la obra, se presentaron variantes en las que se empleaban tablestacas metálicas y rellenos formando dique o escollera.

Los cilindros pretensados constituyen la estructura principal de la obra, tienen un diámetro exterior de $1,40 \mathrm{~m}$ y $12 \mathrm{~cm}$ de espesor. Este tipo de cilindro, hueco, es de empleo corriente, y se fabrican en trozos de $4,90 \mathrm{~m}$ de longitud, utilizando máquinas centrifugadoras alimentadas con hormigón denso y suficientemente seco para poder desencofrar unas tres horas después de hormigonar. Este hormigón tiene una resistencia cuyo campo se extiende de 350 a $560 \mathrm{~kg} / \mathrm{cm}^{2}$.

En el interior de los encofrados se colocan tubos de goma convenientemente hinchados que sirven de molde para los conductos de los cables del pretensado. Estos núcleos de goma se retiran después de haberse iniciado el fraguado del hormigón.

Para obtener un cilindro de una determinada longitud, basta acoplar los trozos de 4,90 m de longitud, unos a continuación de los otros, para pretensarlos después y constituir un cilindro de la longitud deseada, Obtenido el grado de tesado previsto, se inyecta una lechada de cemento y se retiran los anclajes auxiliares de las extremidades.

Cada cilindro tiene 12 cables para el pretensado, cada uno de estos cables, a su vez, está formado por otros 12 alambres de $4,8 \mathrm{~mm}$, que se tesan hasta conseguir una tensión de $10.546 \mathrm{~kg} / \mathrm{cm}^{2}$.

Los cilindros han sido prefabricados por la Raymond International Inc. en sus talleres de Mandeville, de Louisiana, desde donde se transportaron, por vía marítima, hasta el pie de obra.

Estos cilindros se hincaron sirviéndose de una machina de la casa Raymond, tipo núm. 4/0 de simple acción y con una capacidad de unos $6.700 \mathrm{~kg} / \mathrm{m}$ por golpe. 

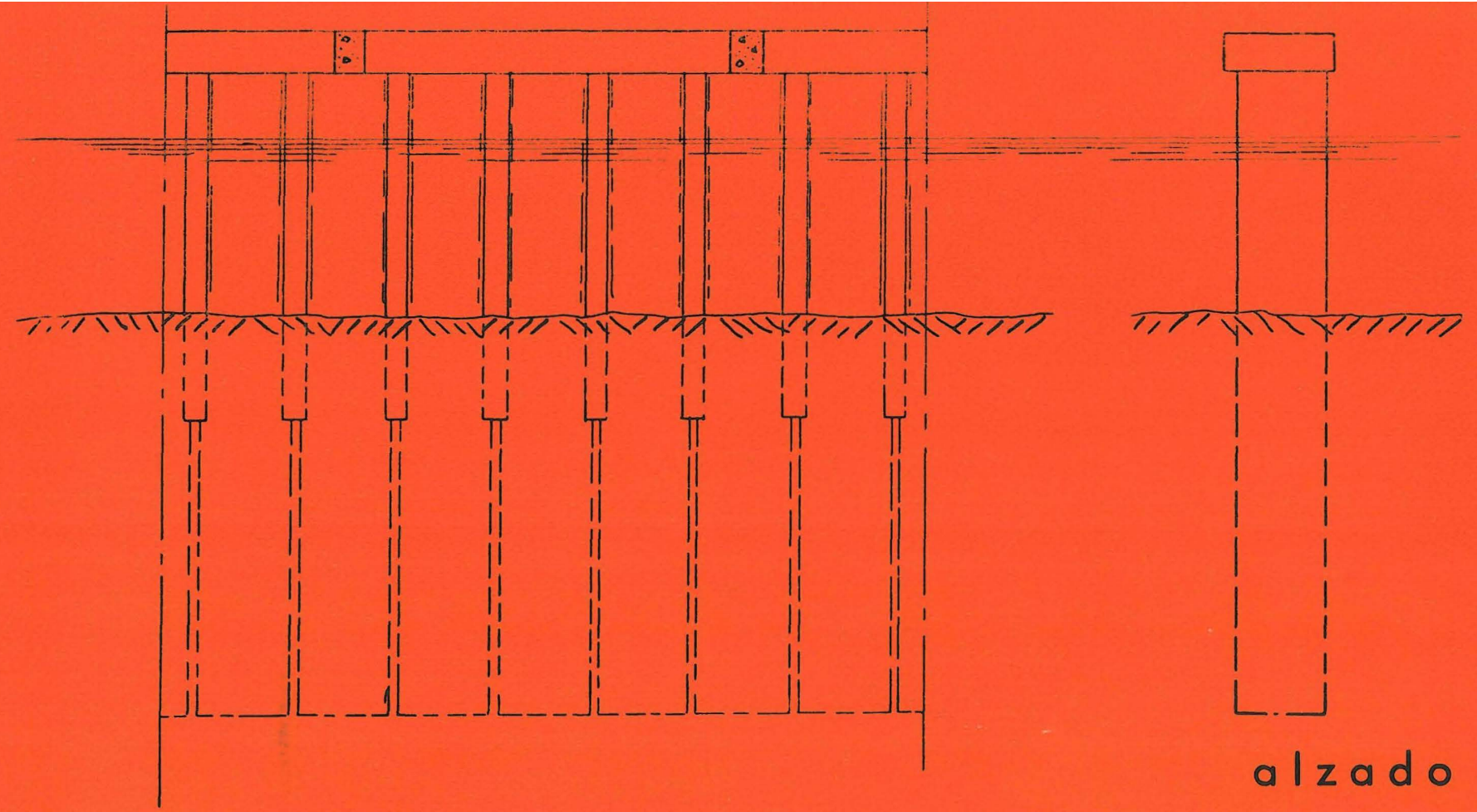

La hinca se realizó espaciando los cilindros a 1,50 m entre ejes, dejando un espacio libre entre cilindro del orden de 15 centímetros.

Los huecos entre pilotes se cierran con tablestacas de $25 \times 25 \mathrm{~cm}$, preparadas para seguir la curvatura de los cilindros. Entre cada hueco formado por dos cilindros consecutivos se colocan dos piezas de madera, una a cada lado, que se aprietan con pernos galvanizados. En ciertos casos, estos huecos se pueden rellenar con hormigón colocado en obra o con piezas prefabricadas con este material.

Antes de colocar la losa de coronación, se rellenan los huecos interiores de los cilindros con arena hasta unos 75 cm de la extremidad superior.

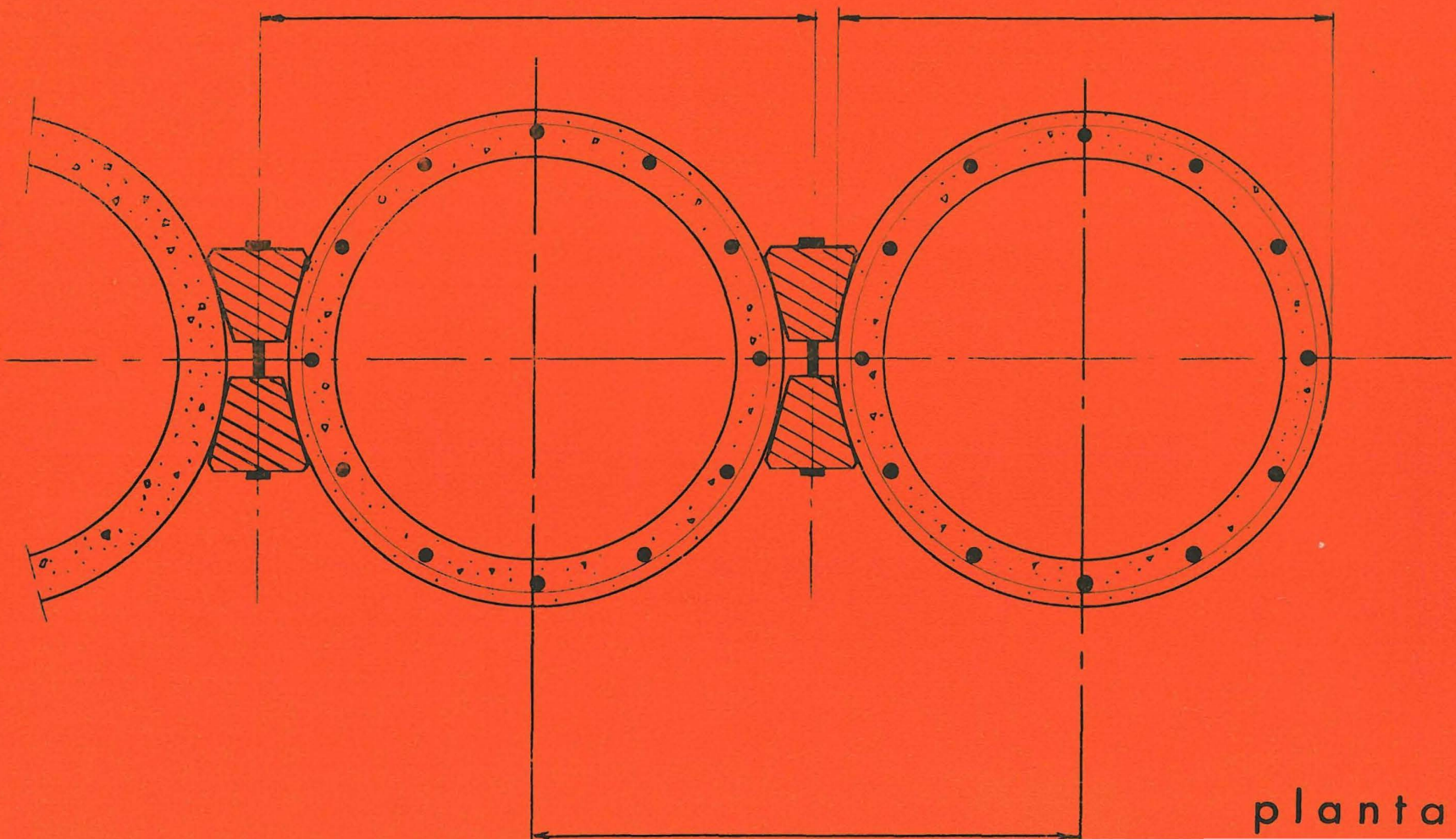




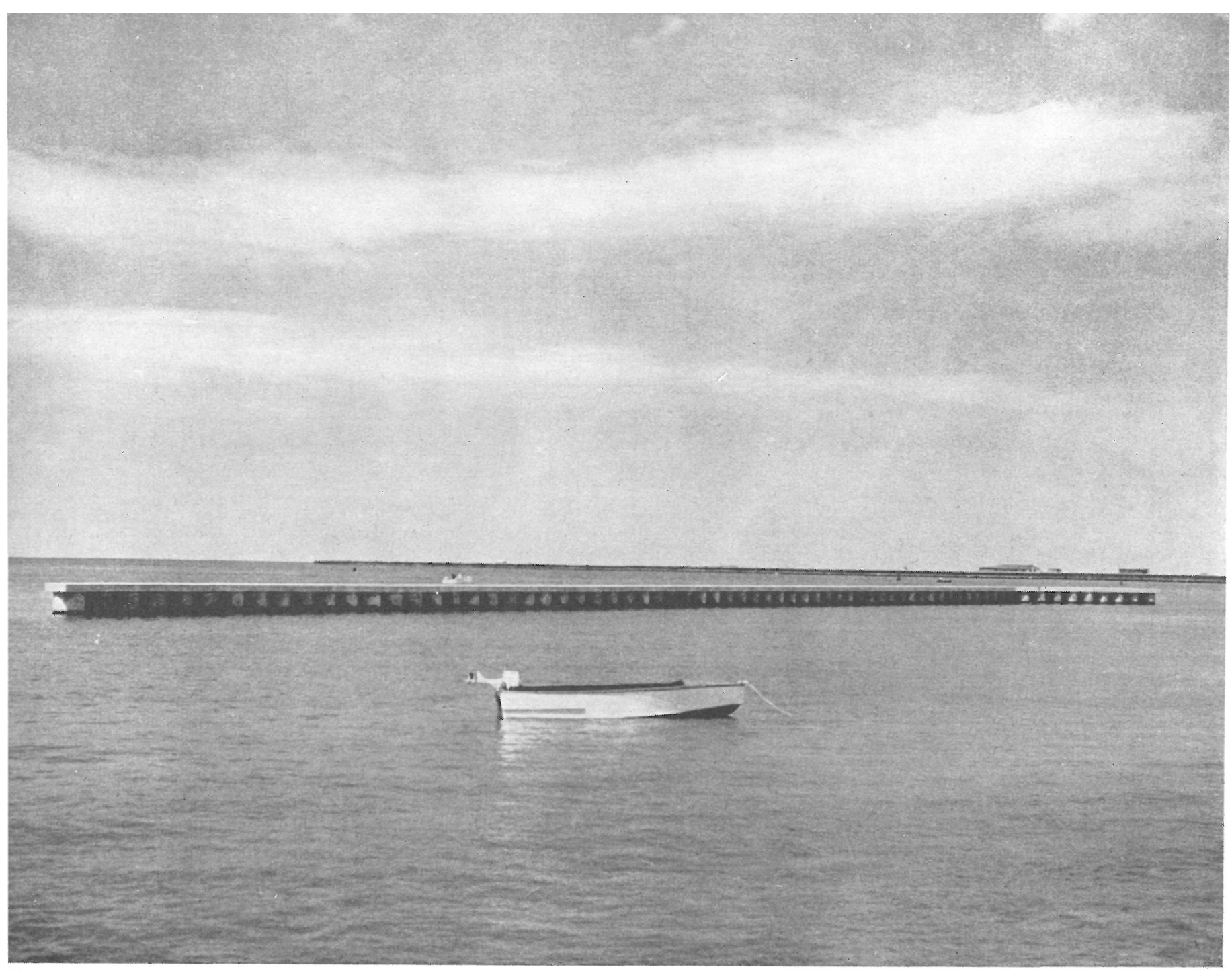

La arena o relleno mejora la rigidez dinámica de los cilindros, y sirve, a su vez, como soporte del hormigón que, a través de los agujeros dejados en la losa de coronación, se va colocando después de situar la referida losa, permitiendo así una trabazón perfecta entre el cilindro y la losa.

La losa de coronación, montada por piezas, se solidariza mediante juntas solapadas y soldadura de sus armaduras, consiguiéndose con esto una gran unión entre ellas y, por ende, una continuidad perfecta a lo largo del muro formado por los cilindros. La losa de coronación lleva un antepecho metálico, el cual permite la circulación del personal sobre el rompeolas y espacio para los pescadores.

En el estudio del rompeolas fueron objeto de consideración las cargas verticales, los efectos erosivos del mar y las condiciones de carga lateral. La carga vertical es del orden de $2.000 \mathrm{~kg}$ por metro. Teniendo en cuenta las condiciones más desfavorables de la localidad, la hinca de los cilindros se llevó a unos $6 \mathrm{~m}$ de profundidad. Con esta penetración, y dadas las condiciones del fondo del mar, la capacidad de carga por cilindro es de unas 51 toneladas, que si se comparan con las 18 que han de soportar, se obtiene un coeficiente de 2,8 para la seguridad de la obra.

En otro rompeolas, próximo al que nos venimos refiriendo, se llevaron las bases de apoyo a $6 \mathrm{~m}$ de profundidad, sin que hasta el momento se haya podido apreciar un efecto erosivo de consideración en estos últimos años. Por todo ello, los efectos de la erosión no fueron causa de inquietud en el estudio del proyecto.

Las condiciones de carga lateral están gobernadas por las olas máximas que se presume han de resistir. Las olas máximas previstas, de acuerdo con la experiencia en esta parte del Pacífico, son de unos 2,40 m de altura, Empleando el método de Sainflow para el análisis de esfuerzos desarrollados por el oleaje, el esfuerzo lateral correspondiente a cada cilindro es de $8.600 \mathrm{~kg}$. La presión ejercida por el suelo sobre cada uno de los cilindros es de $48.175 \mathrm{~kg}$, lo a cada cilindro es de $8.600 \mathrm{~kg}$. La presión ejercida por el suelo sobre cada uno de los cilindros es de $48.175 \mathrm{~kg}$, lo
que supone un coeficiente de seguridad de 5,6. Este coeficiente no se consideró excesivamente elevado, ya que en el proyecto se incluyen ciertas hipótesis y dudas, así como otras consideraciones que no se han tenido en cuenta. Todo esto justifica el que no se haya hecho una reducción importante en la profundidad de hinca de los cilindros.

El rompeolas de Christian se halla dentro del agua hasta unos $4 \mathrm{~m}$ de profundidad, y la parte superior o losa se encuentra a unos $3,50 \mathrm{~m}$ sobre el nivel del mar. Los sondeos de reconocimiento cortaron una capa fangosa de 0,30 a $0,90 \mathrm{~m}$ de espesor, a la que sigue otra de potencia media de 7,60 $\mathrm{m}$ con formación de arena.

La longitud del rompeolas es de $300 \mathrm{~m}$, y salió a subasta previendo una estructura celular cilíndrica cuya altura total a partir de cimientos sería de 12 metros. 